\title{
Whipple's Pancreatoduodenectomy for Periampullary Carcinoma in a HIV Positive Patient
}

\author{
Supriyo Ghatak • Pankaj Kr Sonar • Somak Das • \\ Avik Bhattacharyya
}

Received: 22 June 2012 / Accepted: 3 September 2012 /Published online: 15 September 2012

(C) Association of Surgeons of India 2012

Screening for HIV infection before surgery is controversial. Many surgical societies and countries recommend screening, mostly for subgroups in a population [1]. Here we present a case of periampullary carcinoma, who was detected to be HIV positive on screening before surgery and underwent successful resection.

A 44-year-old Indian woman presented with jaundice for 6 months. Her serum bilirubin was $2.5 / 1.9 \mathrm{mg} / \mathrm{dl}$ (total/conjugated), alkaline phosphatase was $307 \mathrm{IU} / \mathrm{dl}$ and CA 19-9 was $25.5 \mathrm{U} / \mathrm{ml}$. She was found to be HIV positive during work up in another hospital. Her CD 4 count was 604 cells $/ \mathrm{ml}$. CT scan showed an ill-defined periampullary tumor. She developed isoflurane induced refractory hypotension on her first visit to operation room. After cardiac re-evaluation she was operated with using sevoflurane as anesthetic agent. Classical Whipple's pancreatoduodenectomy and feeding jejunostomy were done. Postoperatively, except for delayed gastric emptying, she recovered well, her nasogastric tube was removed on day 9 , and she was discharged on day 11 . Her biopsy report showed a 2-cm well-differentiated papillary adenocarcinoma of periampullary region with no perineural/lymphovascular invasion. Her retropancreatic margin, bile duct margin and stomach margin were free of tumor. Peripancreatic (4), common hepatic artery (1) and aortocaval lymphnodes (4) were also free of tumor. She was doing well 3 months after surgery.

The manuscript has not been presented in any meeting.

S. Ghatak $(\varangle) \cdot$ P. K. Sonar $\cdot$ S. Das

Division of Surgical Gastroenterology, School of Digestive \&

Liver Diseases, IPGME \& R, SSKM Hospitals,

244, A J C Bose Road,

Kolkata 700020, India

e-mail: drsupriyo@yahoo.co.in

A. Bhattacharyya

Division of GI Radiology, School of Digestive \& Liver Diseases,

IPGME \& R, SSKM Hospitals,

244, A J C Bose Road,

Kolkata 700020, India
Although controversial, many countries recommend screening for HIV before surgery, especially in high-risk population. There are arguments against routine screening, like mental trauma and social ostracism. But at the same time, the universal precaution for all patients is not practically feasible in almost all government hospitals and most of the private hospitals in India. As the risk to the health care provider and patient's spouse is real and the treatment provided in our country for HIV is free, there may be justification in routine screening for all patients undergoing surgery.

The influence of surgery on the course of HIV or vice versa is not clear for periampullary cancer. Literature search (English language, Pubmed) revealed only one case of pancreatoduodenectomy for periampullary carcinoma reported from Switzerland with good outcome [2]. CD4 count of less than 200 cells $/ \mathrm{ml}$ is considered to be a predictor for complications for HIV patients undergoing surgery, but other reports suggest that there is no correlation between CD 4 count and operative complications $[2,3]$.

This case supports the view that major abdominal surgery like pancreatoduodenectomy can be undertaken with good outcome in the HIV positive patient.

Conflict of interest there is no conflict of interest. The authors do not have any association with any pharmaceutical or equipment company and they have not availed of any grant provided by any source.

\section{References}

1. Li L, Wu Z, Sheng Wu S et al (2007) Mandatory HIV testing in China: the perception of health-care providers. Int J STD AIDS 18:476-481

2. Wildi S, Büchler MW, Eigenmann F et al (2001) Pancreatic resection for cancer in a young patient with HIV infection. Dig Surg 18:327-328

3. Guth AA, Hofstetter SR, Pachter HL (1996) Human immunodeficiency virus and the trauma patient: factors influencing postoperative infectious complications. J Trauma 41:251-255 